\title{
Inhomogeneous behavior of anti-plane shear crack in softening material with local unloading
}

\author{
T.H. HaO \\ X.T. Zhang \\ Instime of Mechanics, Academia Sinica. Beimmg lomso. Proples Republic of Chinsi \\ K.C. Hwang

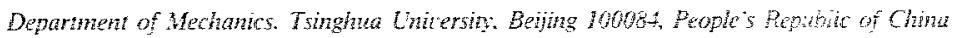

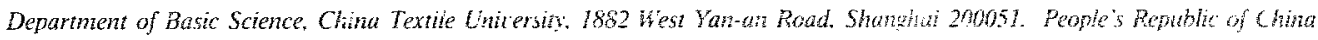

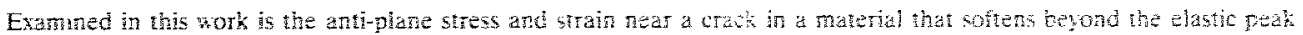

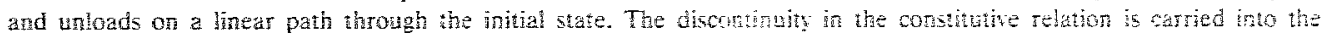

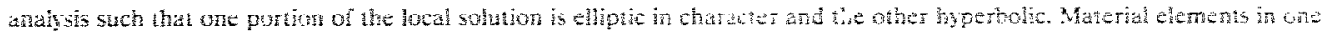

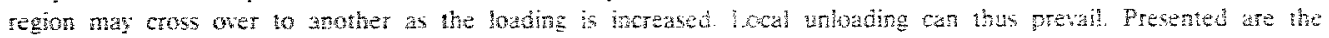

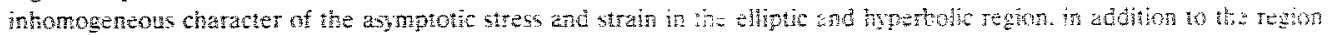

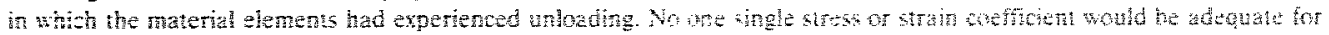
describing etack instabints.
\end{abstract}

\section{Introduction}

A material is said to suffer permanent deformuton or damage when it surpasses the alastic limit or yield poin. Thereafter. the stress and stran response may be noninear and unoading would follow a different pats. It is customary to pre-assign the same constimuive ralation for ach ofement in the stetern.

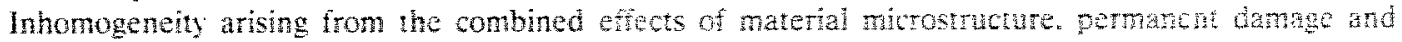
loading is nomally neglected. Much of the a prori assumptions may apeat to be reashathe tor the

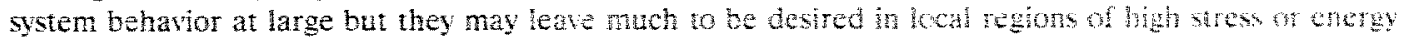
gradients such as a line of discontinuty of orack.

What can be demonstrated from the ciassical heory of plastictis is the lact of thingmogentive of the

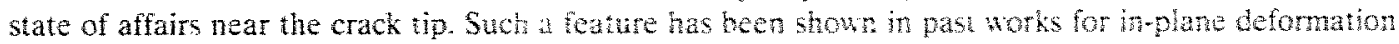
$[1-3]$ and anti-plane shear [4,5]. The charaet of inhomogeneity depends directy on tye assumed form of the constitutive equation. This is reflected in the result of this wot in contrast to that in [5] although both dealt with sofening materials. Oner works related to material danage and softening can bo found in $[6,7]$. One way of not comminting to a particular form of the constitutive relation is to provide a scheme where the stress and stan response of each material clemen is derived [3] rather than preassigned.

\section{Basic equations}

Under anti-plane shear, all material elements are assumed to deform out of the $x_{1} x_{2}$-plane with $i i_{3}$ along the $x_{3}$-axis being the only non-zero displacement component. Accordingly, only two stress components $\tau_{13}$ and $\tau_{23}$ prevail with the corresponding strains $\gamma_{13}$ and $\gamma_{23}$. The relations between $\gamma_{i j}$ and $u_{3}$ are:

$$
\gamma_{13}=\frac{\partial u_{3}}{\partial x_{1}}, \quad \gamma_{33}=\frac{\partial u_{3}}{\partial x_{2}}
$$




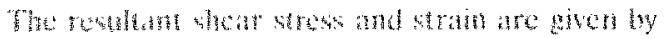

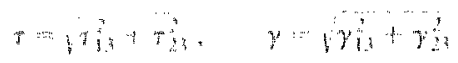

The :sumption of propmitomal wading then yiclds

$$
\tau_{1,1}=\frac{\tau}{\gamma} \gamma_{1,3}, \quad \tau_{23}=\frac{\tau}{\gamma} \gamma_{23}
$$

such that $\tau_{i j}$ and $\gamma_{i j}$ depend only on the space variables $x,(j=1,2)$. Equilibrium is enforced by

$$
\frac{\partial \tau_{13}}{\partial x_{1}}+\frac{\partial \tau_{23}}{\partial x_{2}}=0
$$

while compatibility is satisfied if

$$
\frac{\partial \gamma_{23}}{\partial x_{1}}-\frac{\partial \gamma_{13}}{\partial x_{2}}=0
$$

Solution to a particular problem requires the a priori assumption of the constitutive relation and boundary conditions for a specific geometry and loading.

\subsection{Transformation to strain plane}

Introduce the function $\psi$ in the complex plane $\gamma_{13}+\mathrm{i} \gamma_{23}$ such that eq. (5) is automatically satisfied:

$$
x_{1}=\frac{\partial \psi}{\partial \gamma_{13}}, \quad x_{2}=\frac{\partial \psi}{\partial \gamma_{23}}
$$

Here, $x_{j}$ are regarded as the dependent variables while $\gamma_{i j}$ are independent:

$$
\gamma_{13}+\mathrm{i} \gamma_{23}=\gamma \mathrm{e}^{\mathrm{i} \phi}
$$

with $\phi$ being the argument of $\gamma$. Alternatively, $x_{j}$ can be expressed in terms of $\gamma$ and $\phi$ as

$$
x_{1}=\cos \phi \frac{\partial \psi}{\partial \gamma}-\frac{\sin \phi}{\gamma} \frac{\partial \psi}{\partial \phi}, \quad x_{2}=\sin \phi \frac{\partial \psi}{\partial \gamma}+\frac{\cos \phi}{\gamma} \frac{\partial \psi}{\partial \phi}
$$

Because of proportional loading, the stress components can also be written as

$$
\tau_{13}+\mathrm{i} \tau_{23}=\tau \mathrm{e}^{\mathrm{i} \phi}
$$

Application of eq. (4) therefore yields

$$
\frac{\tau(\gamma)}{\gamma \tau^{\prime}(\gamma)} \frac{\partial^{2} \psi}{\partial \gamma^{2}}+\frac{1}{\gamma} \frac{\partial \psi}{\partial \gamma}+\frac{1}{\gamma^{2}} \frac{\partial^{2} \psi}{\partial \phi^{2}}=0
$$

in which $\tau^{\prime}(\gamma)=\mathrm{d} \tau / \mathrm{d} \gamma$. The anti-plane displacement $u_{3}$ is related to $\psi$ as

$$
u_{3}=\gamma \frac{\partial \psi}{\partial \gamma}-\psi \quad \text { or } \quad \psi=r \frac{\partial u_{3}}{\partial r}-u_{3}
$$

with $r$ being the radial distance in a polar coordinate system of the physical plane $x_{1} x_{2}$.

\subsection{Constitutive relation}

The constitutive relation of the material is displayed graphically in Fig. 1 . It has a discontinuity at $\gamma_{0}$ where the shear $\tau$ attains its maximum:

$$
\tau=G \begin{cases}\gamma, & \text { for } \gamma \leqslant \gamma_{0} \\ \gamma_{0} \sqrt{\frac{\gamma_{0}}{\gamma}}, & \text { for } \gamma \geqslant \gamma_{0}\end{cases}
$$




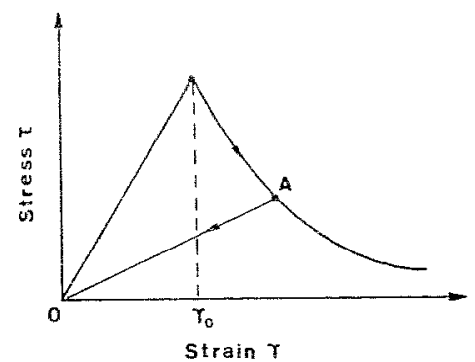

Fig. 1. Stress and strain diagram for material with softening.

Should unloading occur at certain point $\left(r_{a}, \theta_{a}\right)$ in the $x_{1} x_{2}$-plane that corresponds to $\mathrm{A}$ in Fig. 1 , the stress/strain would follow the straight line $\mathrm{A} \rightarrow 0$ with the modulus $G_{i}=\tau_{i} / \gamma_{t}$. Equation (12) would thus give

$$
\frac{G_{t}}{G}=\left(\frac{\gamma_{0}}{\gamma_{t}}\right)^{3 / 2}
$$

\section{Semi-infinite crack: solution form}

Let a semi-infinite crack occupy the ncgative portion of the $x_{1}$-axis as indicated in Fin. 2 . Equations (8) transform the crack in $x_{1}+\mathrm{i} x_{2}$ onto the seminfinite portion of the plane $y_{15}+1 y_{2}$ defined by $0 \leqslant \phi \leqslant \pi$, the limits of which give the principal shear directions that correspond to the lower and upper crack surface, respectively. Note from eqs. (8) that

$$
x_{2}=0 \rightarrow \frac{\partial U}{\partial \theta}=0 \text { at } \phi=0 \text { and } \pi
$$

The strain singularity at $x_{1}=x_{2}=0$ maps 0 infinity in the $y$-plane such that the derivatwes of $f_{y}$ vanish at infinity.

In elastic case, it is known that

$$
x_{1}-\mathrm{i} x_{2}=\mathrm{e}^{\mathrm{i} \dot{\phi}}\left(\frac{\partial \psi}{\partial \gamma}-\frac{1}{\gamma} \frac{\partial \psi}{\partial \phi}\right) \rightarrow-\frac{1}{2 \gamma}\left(\frac{k_{3}}{G}\right)^{2} \mathrm{e}^{-2 i \phi}, \quad \gamma \rightarrow 0
$$

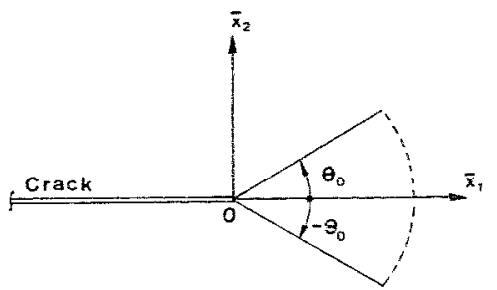

Fig. 2. Semi-infinite crack geometry. 


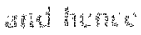

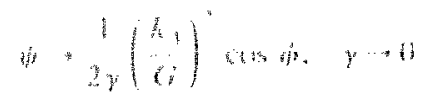

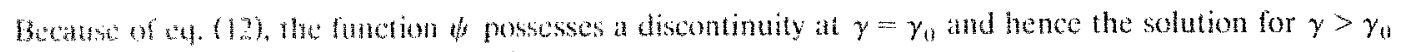
and $y<y_{\text {ta }}$ will be discussed sepurately.

\subsection{Elliptic region $\left(\gamma<\gamma_{0}\right)$}

A form of $\psi$ will he selected such that the free surface crack condition will be satisfied in addition to the gowerning eq. (9). The choice

$$
\psi=\frac{l}{2}\left\{\frac{1}{\gamma}+\gamma\left[2 G \int_{\gamma_{0}}^{\infty} \frac{\mathrm{d} u}{u^{2} \tau(u)}-\gamma_{0}^{-2}\right]\right\} \cos \phi, \quad \gamma<\gamma_{0}
$$

is thus made. The length parameter $l$ stands for

$$
l=\left(\frac{k_{3}}{G}\right)^{2}
$$

with $k_{3}$ being a load intensity factor. It is expedient to introduce the quantities

$$
R(\gamma)=\frac{l}{2} G \cdot \frac{1}{\gamma \tau(\gamma)}, \quad S(\gamma)=\frac{l}{2} G\left[2 \int_{\gamma}^{\infty} \frac{\mathrm{d} u}{u^{2} \tau(u)}-\frac{1}{\gamma \tau(\gamma)}\right]
$$

It follows from eqs. (8) that

$$
x_{1}=S\left(\gamma_{0}\right)-R(\gamma) \cos 2 \phi, \quad x_{2}=-R(\gamma) \sin 2 \phi, \quad \gamma<\gamma_{0}
$$

in which

$$
R(\gamma)=\frac{l}{2 \gamma^{2}}
$$

because of eq. (12). A system of polar coordinates $\bar{r}_{1}=r_{1} / l$ and $\theta_{1}$ in dimensionless form can thus be introduced:

$$
\bar{x}_{1}=\frac{3}{2 \gamma_{0}^{2}}+\bar{r}_{1} \cos \theta_{1}=\frac{3}{2 \gamma_{0}^{2}}-\frac{1}{2 \gamma^{2}} \cos 2 \phi, \quad \bar{x}_{2}=\bar{r}_{1} \sin \theta_{1}=-\frac{1}{2 \gamma^{2}} \sin 2 \phi, \quad \gamma<\gamma_{0}
$$

where $\bar{x}_{i}=x_{j} / l$. This leads to

$$
\gamma=\frac{1}{\sqrt{2 \bar{r}_{1}}}, \quad \phi=\frac{1}{2}\left(\theta_{1}+\pi\right)
$$

such that

$$
\vec{r}_{1}^{2}=\left(\bar{x}_{1}^{2}-\frac{3}{2 \gamma_{0}^{2}}\right)^{2}+\bar{x}_{2}^{2}, \quad \tan \theta_{1}=\frac{\bar{x}_{2}}{\bar{x}_{1}-\frac{3}{2 \gamma_{0}^{2}}}
$$

Refer to Fig. 3. The iegion $\gamma<\gamma_{0}$ would correspond to that outside the circle of radius $1 / 2 \gamma_{0}^{2}$ with center at $\bar{x}_{1}=3 / 2 \gamma_{0}^{2}$ and $\bar{x}_{2}=0$ as given in Fig. 3. It can be seen from eqs. (22) that

$$
\left(\bar{x}_{1}-\frac{3}{2 \gamma_{0}^{2}}\right)^{2}+\bar{x}_{2}^{2}=\frac{1}{4 \gamma_{0}^{4}}
$$




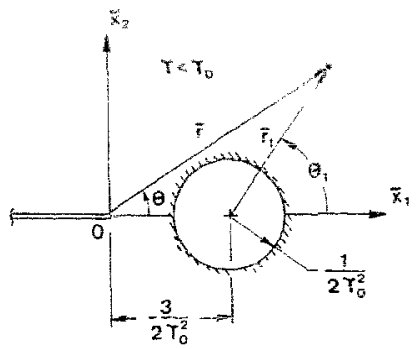

Fig. 3. Elliptic region outside circle.

3.2. Hyperbolic region $\left(\gamma>\gamma_{0}\right)$

When $\mathrm{d} \tau / \mathrm{d} \gamma<0$ or $\gamma>\gamma_{0}$, eq. (10) is hyperbolic and hence the choice

$$
\psi=l G\left[\gamma \int_{\gamma}^{\infty} \frac{\mathrm{d} u}{u^{2} \tau(u)}\right] \cos \phi, \quad \gamma>\gamma_{0}
$$

is made. Refer to the Appendix for the elliptic and hyperbolic character of the governing differential equations. Equations (19) become

$$
x_{1}=S(\gamma)-R(\gamma) \cos 2 \phi, \quad x_{2}=-R(\gamma) \sin 2 \phi, \quad \gamma>\gamma_{0}
$$

For the material at hand, $R$ and $S$ are given by

$$
R(\gamma)=\frac{l}{2} \frac{1}{\gamma_{0} \overline{\gamma \gamma_{0}}}, \quad S(\gamma)=\frac{3 l}{2} \frac{1}{\gamma_{0 l} \overline{\gamma \gamma_{0}}} . \quad y>\gamma_{0}
$$

Referring to Fig. 3, the coordinates $(\bar{r}, \theta)$ are

$$
\bar{x}_{1}=\bar{r} \cos \theta=\frac{1}{2 \gamma_{0} \gamma \gamma_{0}}(3-\cos 2 \phi), \quad \bar{x}_{2}=\bar{r} \sin \theta=-\frac{1}{2 \gamma_{0} \hat{\gamma} \gamma_{0}} \sin 2 \phi, \quad y>\gamma_{i}
$$

which can be used to give

$$
\sqrt{\gamma}=\frac{1}{2 \bar{r} \gamma_{0}, \sqrt{\gamma_{0}}}[3 \cos \theta \pm T(\theta)], \quad \gamma>\gamma_{0}
$$

provided that

$$
T(\theta)=\sqrt{1-9 \sin ^{2} \theta}
$$

Equations (29) to (31) can be further combined to obtain

$$
\begin{aligned}
& \cos \phi=-2 \sqrt{2} \sin \theta\left[1+3 \sin ^{2} \theta \pm T(\theta) \cos \theta\right]^{-1 / 2} \\
& \sin \phi=\frac{1}{\sqrt{2}}[\cos \theta \pm T(\theta)]\left[1+3 \sin ^{2} \theta \pm T(\theta) \cos \theta\right]^{-1 / 2}, \quad \gamma>\gamma_{0}
\end{aligned}
$$

The value of $\sqrt{\gamma}$ in eqs. (30) must be real and positive and this prevails if and only if

$$
|\cos \theta| \geqslant \frac{2 \sqrt{2}}{3}, \text { for }-\theta_{0} \leqslant \theta \leqslant \theta_{0}
$$




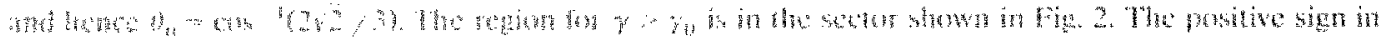

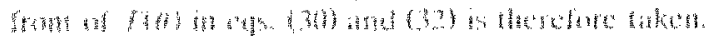

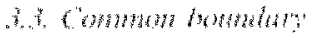

The region in which $\mathrm{d} t / \mathrm{d} \gamma<0\left(\gamma>\gamma_{0}\right)$ and $\mathrm{d} r / \mathrm{d} \gamma\left(\gamma<\gamma_{0}\right)$ meet at a common boundary. It can be found from the continuity of displacement and traction. Substituting eq. (17) into the first of eqs. (11) gives

$$
\vec{\pi}_{3}=\frac{u_{3}}{l}=-\frac{1}{\gamma} \cos \phi, \quad \gamma<\gamma_{0}
$$

while eqs. (26) and (11) render

$$
\bar{u}_{3}=\frac{u_{3}}{l}=-\frac{1}{\gamma_{0}} \sqrt{\frac{\bar{\gamma}}{\gamma_{0}}} \cos \phi, \quad \gamma>\gamma_{0}
$$

Equations (23) and (34) can be combined:

$$
\bar{u}_{3}=\frac{1}{\sqrt{2 \bar{r}_{1}}} \sin \left(\frac{\theta_{1}}{2}\right), \quad \gamma<\gamma_{0}
$$

while eqs. (30), (32) and (35) yield

$$
\bar{u}_{3}=\frac{1}{\sqrt{2} \bar{r} \gamma_{0}^{3}} \sin \theta \cdot \frac{[3 \cos \theta+T(\theta)]^{3 / 2}}{[\cos \theta+T(\theta)]^{1 / 2}}, \quad \gamma>\gamma_{0}
$$

The common boundary that is common to the elliptic and hyperbolic region is obtained by equating eqs. (36) and (37):

$$
\overline{2} \sqrt{2 \bar{r}_{1}} \sin \frac{\theta}{2}=f(\theta)
$$

in which

$$
f(\theta)=\frac{1}{\sqrt{2} \gamma_{0}^{3}} \sin \theta \cdot \frac{[3 \cos \theta+T(\theta)]^{3 / 2}}{[\cos \theta+T(\theta)]^{1 / 2}}
$$

It can be shown that the common boundary in terms of $\vec{r}$ and $\theta$ is given by

$$
\bar{r}^{6} \sin ^{2} \theta+2 \bar{r}^{3} f^{2} \cos \theta+\frac{3}{\left(\gamma \gamma_{0}\right)^{2}} f^{2}-f^{4}=0
$$

The continuity of tractions is satisfied. Knowing that $\bar{r}=r / l$ and as $\theta \rightarrow 0$

$$
r \rightarrow \frac{4}{\sqrt{3}} \frac{l}{\gamma_{0}^{2}} \sin \theta, \text { on } \Gamma
$$

where $\Gamma$ denotes the common boundary in Fig. 4. It moves as the load factor $l=\left(k_{3} / G\right)^{2}$ is increased. A point such as $p_{1}$ inside $\Gamma_{1}$ or the hyperbolic region at one instance $t_{1}$ would be left behind as $\Gamma_{1}$ advances forward to $\Gamma_{\mathrm{L}}$, at a time $t_{2}>t_{\mathrm{1}}$. Unloading would thus take place along a line such as AO in Fig. 1 with decreasing $\gamma$. The point $p_{1}$ would eventually enter into the elliptic regiun when the threshold $\left(r_{0}, \theta_{0}\right)$ is surpassed. 


\section{Unhading region}

II is obvious from Fig. 4 that under increasing load, every etement passes ingough the hyperboh region $|\theta|<\theta_{0}$. Equation (36) yields $\vec{u}_{3}$ in the elliptic region:

$$
\bar{u}_{3}=\frac{\sqrt{3}}{\gamma_{0}}-\frac{\gamma_{0}}{\sqrt{3}} \bar{r} \cos \theta+\mathrm{O}\left(\bar{r}^{2}\right), \quad \gamma<\gamma_{0}
$$

and eq. (37) gives $\bar{u}_{3}$ in the hyperbolic region:

$$
\vec{u}_{3}=\frac{1}{\bar{r}}\left[g(\theta)+\mathrm{O}\left(\theta^{3}\right)\right], \quad \gamma>\gamma_{0}
$$

where $g(\theta)=4 \theta / \gamma_{0}^{3}$. The unloading characteristics can be summarized as:

- A hyperbolic region advances and leaves behind a region in which the elements undergo unloading. That is, when a point, say $\left(r_{\mathrm{a}}, \theta_{\mathrm{a}}\right)$ at $\mathrm{A}$ in Fig. 1 , passes by $\left(r_{0}, \theta_{0}\right)$ with increasing load.

- The modulus of this unloaded region depends on the strain $\gamma_{i}$ us the puint where unloading started. Refer to eq. (12).

- The interface between the unloaded and elliptic region is a straight line tangent to the circle given by eq. (24).

\subsection{Unloaded / hyperbolic interface}

Suppose that $\bar{r}=h(\theta)$ describes the interface of the unloaded and hyperbolic region. If untording starts at the point $\left(r_{\mathrm{a}}, \theta_{\mathrm{a}}\right)$, then

$$
l=\frac{r}{h(\theta)}
$$

Recall from eq. (43) that

$$
u_{3}=\frac{l^{2}}{r^{2}} g(\theta)
$$

Moreover, eqs. (1) and (2) can be combines

$$
y=\sqrt{\left(\frac{\partial u_{3}}{\partial r}\right)^{2}+\frac{1}{r^{2}}\left(\frac{\partial u_{3}}{\partial \theta}\right)^{2}}
$$

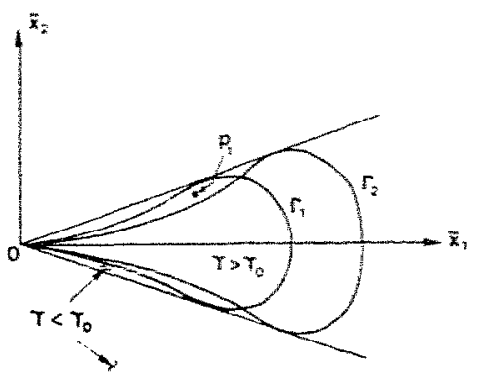

Fig. 4. Common boundary noving with incteasing load. 
With $\gamma_{i}$ being the strain at $\left(\gamma_{i 2}, \theta_{i}\right)$ and using eq. (44), there results

$$
\gamma_{1}=\frac{1}{t^{2}(\theta)} \lg ^{2}(\theta)+g^{2}(\theta)
$$

where $\dot{g}=\mathrm{d} g / \mathrm{d} \theta$. Equation (47) may be inserted into (13) to give

$$
G_{r}=\frac{G}{\gamma_{0 !} \overline{\gamma_{0}}}\left[\dot{g}^{2}(\theta)+g^{2}(\theta)\right]^{-3 / 4} h^{3}(\theta)
$$

Equation (48) applies to any point in the unloading region.

To find $u_{3}$, use will be made of the equation of equilibrium:

$$
\frac{1}{\bar{r}} \frac{\partial}{\partial \bar{r}}\left[G_{t} \bar{r} \frac{\partial \bar{u}_{3}}{\partial \bar{r}}\right]+\frac{1}{\bar{r}^{2}} \frac{\partial}{\partial \theta}\left[G_{t} \frac{\partial \bar{u}_{3}}{\partial \theta}\right]=0
$$

A solution of $\vec{u}_{3}$ is in the form

$$
\bar{u}_{3}=w_{0}+\sum_{m=1}^{\infty} \bar{r}^{m} w_{m}(\theta)
$$

with $w_{0}$ being a constant. Let

$$
h(\theta)=h_{0} \theta^{n}+\mathrm{O}\left(\theta^{n}\right)
$$

Equation (51) may be put into eq. (48). This gives

$$
G_{t} \rightarrow \frac{1}{8} G h_{0}^{3} \gamma_{0}^{3} \theta^{3 n}, \text { as } \theta \rightarrow 0
$$

It can be shown by substituting eqs. (52) and (50) into eq. (49) that

$$
\theta \frac{\mathrm{d}^{2} w_{k}}{\mathrm{~d} \theta^{2}}+3 n \frac{\mathrm{d} w_{k}}{\mathrm{~d} \theta}+m^{2} \theta w_{k}=0
$$

This is a second order ordinary linear differential equation with variable coefficients. The solution is [8]

$$
w_{m}(\theta)=c_{0} \theta^{1-3 n}+c_{1}
$$

for $m=0$. Two linearly independent solutions prevail for $m \neq 0$ :

$$
w_{m}=a_{m}\left[1-\frac{m^{2}}{2(1+3 n)} \theta^{2}+\cdots\right], \quad w_{m}=b_{m}\left[\theta^{1-3 n}+\frac{m^{2}}{6(n-1)} \theta^{3(1-n)}+\cdots\right]
$$

in which $a_{m}$ and $b_{m}$ are arbitrary constants. In the limit as $\theta \rightarrow 0$, only the leading terms need to be retained:

$$
w_{m} \rightarrow a_{m}+b_{m} \theta^{1-3 n}, \text { as } \theta \rightarrow 0
$$

If the bracket notation []$_{u / k}$ is used to denote the difference of the quantity inside across the interface of the unloaded and hyperbolic region as

$$
[]_{u / h}=[]_{u}-[]_{h}
$$

then

$$
\left[\bar{u}_{3}\right]_{u / h}=\left[\frac{\partial \bar{u}_{3}}{\partial \theta}\right]_{u / h}=0
$$


Equations (44) and (51) may be first combined to obtain

$$
\ddot{r}=h_{0} \theta^{n}+O\left(\theta^{n}\right) \rightarrow \theta-\left(\frac{\bar{r}}{A}\right)^{1 / n}
$$

On the hyperbolic side of the interface, eq. (43) may be used:

$$
\left[\bar{u}_{3}\right]_{h}=\frac{4 \theta}{\bar{r} \gamma_{0}^{3}}+\cdots \rightarrow \frac{4}{\gamma_{0}^{3} h_{0}^{1 / n}} \bar{r}^{\frac{1}{n}-1}+\cdots, \quad\left[\frac{\partial \bar{u}_{3}}{\partial \theta}\right]_{h} \rightarrow \frac{4}{\gamma_{n}^{3}} \frac{1}{\bar{\gamma}}+\cdots, \quad \gamma>\gamma_{0}
$$

Equation (50) gives $\bar{u}_{3}$ and $\partial \bar{u}_{3} / \partial \theta$ for the unloaded side:

$$
\begin{aligned}
& {\left[\bar{u}_{3}\right]_{u}=w_{0}+\sum_{m=1}^{\infty} \bar{r}^{m}\left(a_{m}+b_{m} \theta^{1-3 n}\right)+\cdots=w_{0}+a_{m} \bar{F}^{m}+b_{m} h_{0}^{3-\frac{1}{n} \bar{r}^{m-j}+\frac{1}{n}}} \\
& {\left[\frac{\partial \bar{u}_{3}}{\partial \theta}\right]_{u}=(1-3 n) b_{m} \bar{r}^{m} \theta^{-3 n}+\cdots=(1-3 n) b_{m} h_{i}^{3} \bar{r}^{m-3}+\cdots}
\end{aligned}
$$

The conditions in eqs. (57) may be imposed on eqs. (59) and (60); they give

$$
\frac{4}{\gamma_{0}^{3} A^{1 / n}} \vec{r}^{\frac{1}{n}-1}=w_{n}+b_{m} h_{0}^{3-\frac{1}{n}} \bar{r}^{n+3-\frac{1}{n}}, \quad \frac{4}{\gamma^{n}} \bar{r}^{-1}=(1-3 n) h_{m=1}^{3} \sum_{m=1}^{\infty} b_{m} \bar{r}^{m-3}
$$

It follows that

$$
n=1, \quad w_{0}=\frac{4}{h_{0} \gamma_{0}^{3}}, \quad b_{1}=0, \quad b_{2}=\frac{1}{\left(h_{0} \gamma_{0}\right)^{3}}
$$

The anti-plane displacement in the unloaded region is thus given by

$$
\begin{aligned}
\bar{u}_{3} & =w_{0}+\bar{r}\left(a_{1}-\frac{1}{8} a_{1} \theta^{2}+\cdots\right)+\vec{r}^{2}\left(a_{2}+\cdots+b_{2} \theta^{-2}+\cdots\right)+\cdots \text { as } \theta \rightarrow 0 \\
& =w_{0}+\bar{r} w_{1}(\theta)+\bar{r}^{2} w_{2}(\theta)+\cdots
\end{aligned}
$$

\subsection{Unloaded / elliptic interface}

Required on the interface $\theta_{0}=|0|$ of the unloaded and elliptic region are the conditions

$$
\left[\bar{u}_{3}\right]_{u / \mathrm{e}}=\left[\frac{\partial \vec{u}_{3}}{\partial \theta}\right]_{u / e}=0
$$

On the side of the elliptic region, eq. (42) prevails while on the unloaded side, eq. (63) applies. These expressions may be substituted in the first of eqs. (64) for $\theta=\theta_{0} \rightarrow 0$ :

$$
w_{0}+\bar{r} w_{1}\left(\theta_{0}\right)+\bar{r}^{2} w_{2}\left(\theta_{0}\right)+\cdots=\frac{\sqrt{3}}{\gamma_{0}}-\frac{\gamma_{0} \bar{r}}{\sqrt{3}} \cos \theta_{0}+\cdots
$$

Equaling coefficients for like power of $\bar{r}, w_{0}$ and $w_{1}\left(\theta_{0}\right)$ are found:

$$
w_{0}=\frac{\sqrt{3}}{\gamma_{0}}, \quad w_{1}\left(\theta_{0}\right)=-\frac{\gamma_{0}}{\sqrt{3}}
$$


The value $n_{0}$ may be used to express $h_{0}$ and $b_{2}$ in eq. (62) in terms of $\gamma_{0}$ :

$$
h_{0}=\frac{2 \sqrt{3}}{\gamma_{0}^{2}}, \quad b_{2}=-\frac{\gamma_{i}^{3}}{12 \sqrt{3}}
$$

The condition $\left[\partial \bar{u}_{3} / \partial \theta\right]_{u / e}$ in eq. (64) would only yield relations for higher order terms and would not be considered.

\section{Summary and discussion of results}

For a material that behaves linearly to a peak and then softens in proportion to the inverse square root of the strain, the stress and strain state near a semi-infinite crack in out-of-plane shear consist of three regions. They could be referred to as the unloaded, hyperbolic and elliptic region. The asymptotic values of $\tau_{i j}$ and $\gamma_{i j}$ are obtained for $G=10^{6} \mathrm{~kg} / \mathrm{cm}^{2}$ and $\gamma_{0}=1$. Remember that $l=\left(k_{3} / G\right)^{2}$ with $k_{3}$ being the elastic Mode III crack-tip stress intensity factor.

\subsection{Unloaded region}

The local strains are

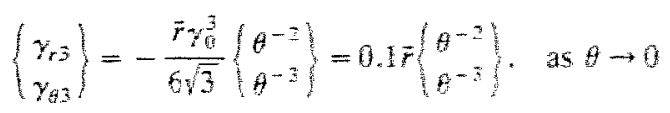

Similarly, the ben streses take the tomms

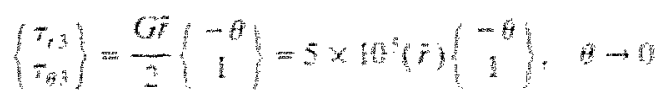

The nodules of the unbated materian is

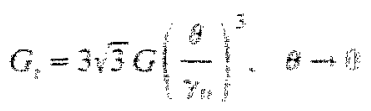

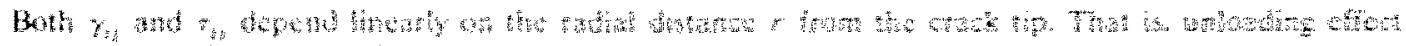

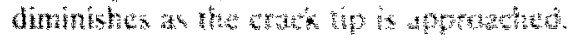

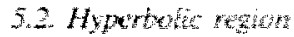

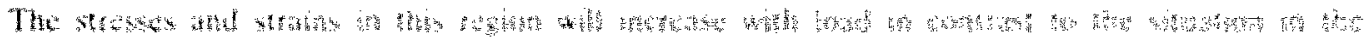

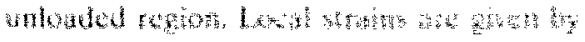

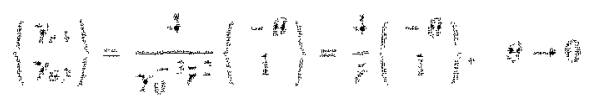

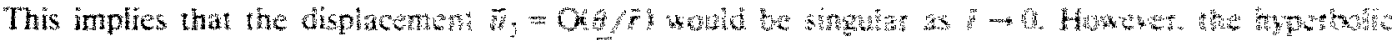

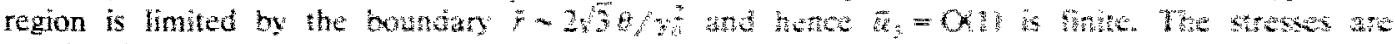
nonsingular:

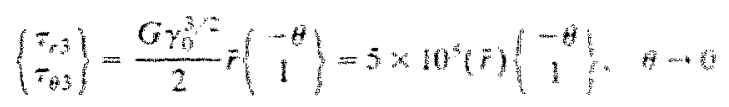

Hence, the stress intensification for a nonlinear material is not the same as that of stran. 


\subsection{Elliptic region}

In the elliptic region, the stresses and strains are independent of the distance fron the crack; they vary only with the angle $\theta$. They are given by

$$
\left\{\begin{array}{l}
\gamma_{r 3} \\
\gamma_{\theta 3}
\end{array}\right\}=\frac{\gamma_{0}}{\sqrt{3}}\left\{\begin{array}{c}
-\cos \theta \\
\sin \theta
\end{array}\right\}=0.58\left(\begin{array}{c}
-\cos \theta \\
\sin \theta
\end{array}\right\}
$$

and

$$
\left\{\begin{array}{l}
\tau_{r 3} \\
\tau_{\theta 3}
\end{array}=\frac{G \gamma_{0}}{\sqrt{3}}\right\}\left(\begin{array}{c}
-\cos \theta \\
\sin \theta
\end{array}\right\}=5.8 \times 10^{5}\left(\begin{array}{c}
-\cos \theta \\
\sin \theta
\end{array}\right\}
$$

\subsection{Concluding remarks}

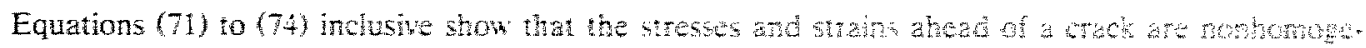

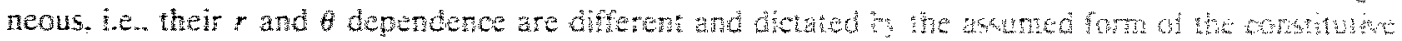

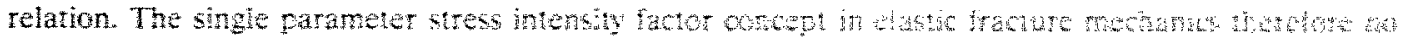

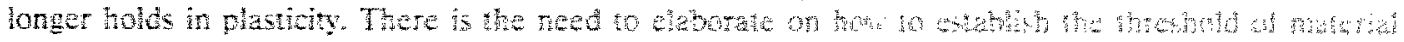

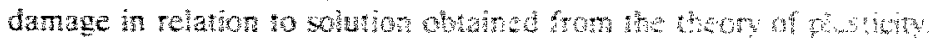

\section{Actontwiedement}

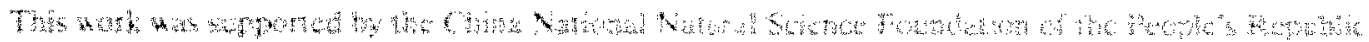

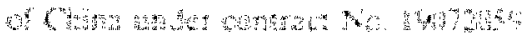

\section{Appendix}

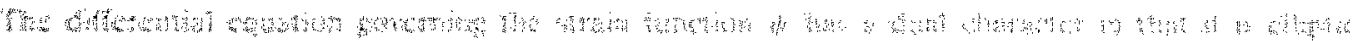

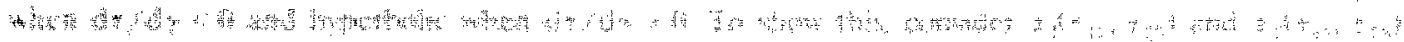

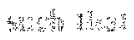

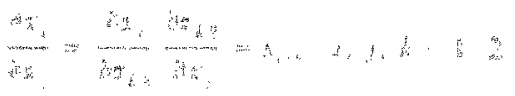

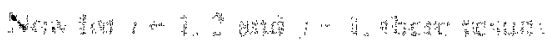

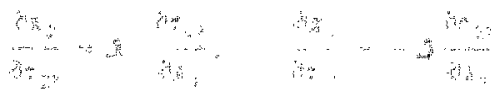

त)

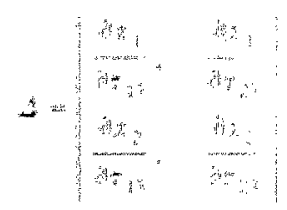

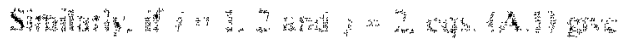

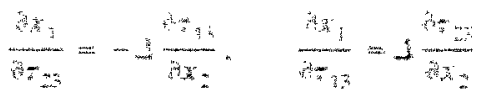


It can be deduced from the first of eqs. (A.2) and second of eqs. (A.4) that

$$
\frac{\partial x_{2}}{\partial \tau_{23}}+\frac{\partial x_{1}}{\partial \tau_{13}}=\Delta\left(\frac{\partial \tau_{13}}{\partial x_{1}}+\frac{\partial \tau_{23}}{\partial x_{2}}\right)=0
$$

as a result of eq. (4) since $\Delta=0$. Equations (6) may be substituted into eq. (A.5) to yield

$$
\frac{\partial}{\partial \tau_{23}}\left(\frac{\partial \psi}{\partial \gamma_{23}}\right)+\frac{\partial}{\partial \tau_{13}}\left(\frac{\partial \psi}{\partial \gamma_{13}}\right)=0
$$

and hence

$$
\frac{\partial \gamma_{j 3}}{\partial \tau_{23}}\left(\frac{\partial^{2} \psi_{i}}{\partial \gamma_{j 3} \partial \gamma_{23}}\right)+\frac{\partial \gamma_{j 3}}{\partial \tau_{13}}\left(\frac{\partial^{2} \psi}{\partial \gamma_{j 3} \partial \gamma_{13}}\right)=0
$$

The property of proportional loading in eq. (3) may be invoked:

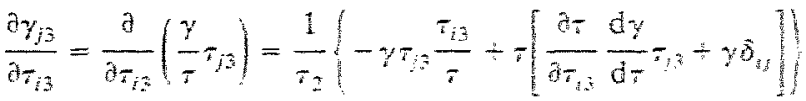

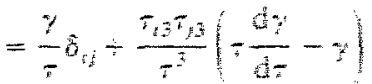

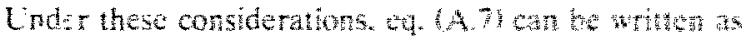

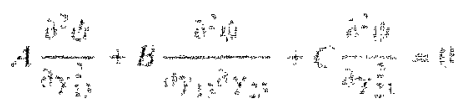

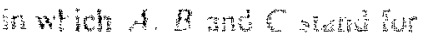

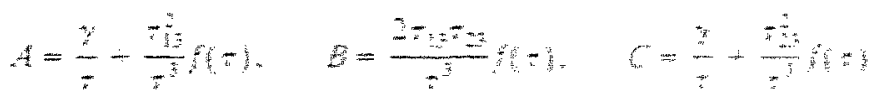

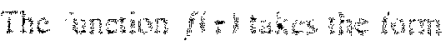

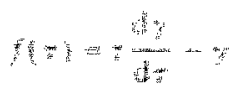

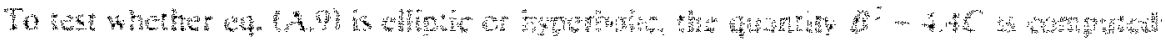

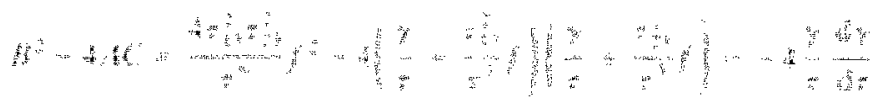

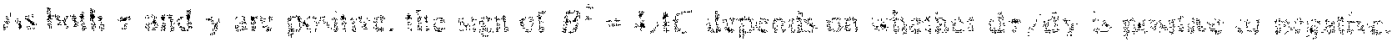

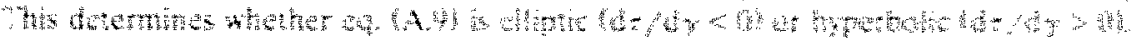

\section{References}

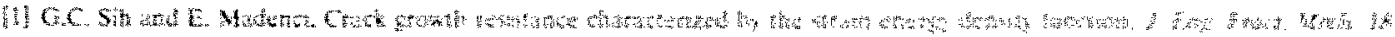
(4)

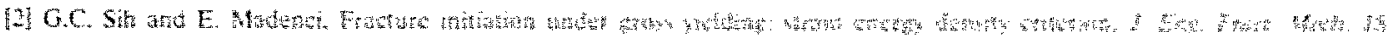
(1983) $607-677$.

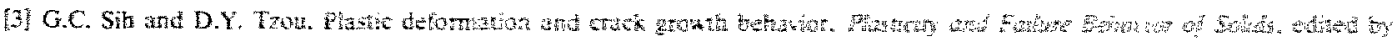

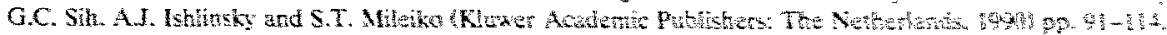


[4] I.K Knowles and E. Sternberg. Discontinuous deformation gradients near the tip of a crack in hits aniplane shear: an example, J. Elasticity 10 (1) (1980) $81-110$.

[5] T.H. Hao, Nonlinear response of antiplane shear crack. Theoret. Appl. Fract. Kech $9(1989) 255-752$.

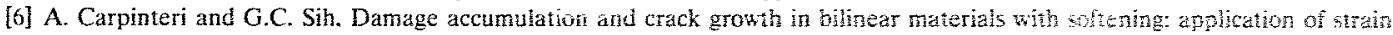
energy density theory. Theoret. Anpl. Fract. Merh. 1 (2) 1984) 145-159

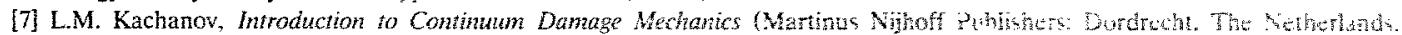
1986). 